\title{
Hemipeneal Morphology of Sri Lankan Dragon Lizards (Sauria: Agamidae)
}

\author{
Kalana Maduwage ${ }^{1,2 *}$ and Anjana Silva ${ }^{2,3}$ \\ ${ }^{1}$ Department of Biochemistry, Faculty of Medicine, University of Peradeniya, Peradeniya, Sri Lanka. \\ ${ }^{2}$ Wildlife Heritage Trust, 1 Lake Crescent, Colombo 2, Sri Lanka. \\ ${ }^{3}$ Department of Parasitology, Faculty of Medicine and Allied Sciences, Rajarata University, \\ Anuradhapura, Sri Lanka. \\ Accepted 18 November 2012
}

\begin{abstract}
The morphology of hemipenes, the male external genitalia of reptiles, is considered to be a character of taxonomic importance. It has, however, until recently been used only rarely in agamid systematics. Phylogenies of Sri Lankan Agamidae, constructed using mitochondrial DNA and hemipeneal morphology, have shown remarkable convergence, highlighting the importance of hemipeneal morphology in the taxonomy of these lizards. Here we present descriptions and illustrations of the hemipenes of 17 of the 18 species of Sri Lankan Agamidae and provide a key to the identification of species groups based on this character.
\end{abstract}

Key words: Calotes, Otocryptis, Sitana, Lyriocephalus, Ceratophora

\section{INTRODUCTION}

Hemipenes, the male external genitalia of reptiles, were first used as a systematic tool by Cope $(1894,1895)$, who noted the systematic value of the structure of this organ. In 1896, Cope used hemipeneal morphology to aid the diagnosis of three species of chameleons. Although many subsequent authors described reptile hemipeneal morphology, the systematic implications were rarely discussed, important exceptions including Wall (1923), Pope and Pope (1933), Smith (1943), and Dowling and Savage (1960).

The first extensive study of the reptile hemipenis was by Cope (1896) who, followed by McCann (1946), provided descriptions of the morphology of some species including Calotes versicolor. It was Dowling and Savage (1960), however, who first defined the hemipeneal characters relevant to systematics and introduced a standard terminology that is still in use.

Branch (1982) reviewed the hemipeneal morphology of platynotan lizards, while Arnold (1986) made a similar study of lacertid lizards; Smith and Fritts (1969) and Presch (1978) of microteiids; Koehler et al., (2012) on anoles (see also Böhme and Ziegler, 2009). Klaver and Böhme (1986) used hemipeneal characters to construct a phylogeny of Chamaeleonidae, establishing the phylogenetic importance of hemipeneal morphology in saurian systematics. In the Agamidae, hemipenes have been rarely described (see Semenov and Dunaev 1989; Baig and Böhme, 1997), though clearly they have value in distinguishing even closely related taxa. For example, Bahir and Silva (2005) used hemipeneal characters to differentiate Otocryptis weigmanni and $O$. nigristigma. Maduwage et al. (2008), showed that phylogenies of the Sri Lankan Agamidae, based on mitochondrial DNA and hemipeneal morphology, show remarkable convergence.

Sri Lanka has a rich agamid fauna, with 15 of its 18 described species being endemic to the island (Bahir and Surasinghe, 2005; Samarawickrama et al., 2006). In addition to Calotes, Otocryptis and Sitana, the island's agamid fauna includes the endemic genera Lyriocephalus, Ceratophora and Cophotis. Here, based on the collections of the Wildlife Heritage Trust of Sri Lanka, we describe and illustrate the hemipeneal morphology of 17 of the 18 species known from the island, the exception being Cophotis dumbara, for which no specimen with averted hemipenes was available.

\section{MATERIALS AND METHODS}

The specimens referred to in this paper are deposited in the collection of the Wildlife Heritage Trust of Sri Lanka (WHT) now housed

*Corresponding author's email: kalanapm@gmail.com 
in the National Museum of Sri Lanka, Colombo (NMSL). All the specimens examined had fully everted hemipenes and were preserved in $70 \%$ alcohol. Hemipeneal morphology was examined using a Motic dissecting stereoscope; drawings were made using a camera-lucida attachment. Snout-vent length (SVL) was measured from tip of snout to base of anal scale using dial-type KWB Vernier callipers, to the nearest $0.1 \mathrm{~mm}$. Terminology of hemipeneal morphology follows Dowling and Savage (1960) except that the hemipenes were everted with their apices posteriorly orientated. Altitudes are given in metres above mean sea level. Geographical coordinates were derived from a Magellan 12channel GPS and inch-to-the-mile topographic maps published by the Survey Department of Sri Lanka.

\section{RESULTS}

\section{Hemipeneal descriptions Otocryptis nigristigma}

Material examined: WHT 6168, $60.3 \mathrm{~mm}$ SVL, Puwakpitiya, Knuckles, $07^{\circ} 34^{\prime} \mathrm{N}, 80^{\circ} 45^{\prime}$ E, alt. $450 \mathrm{~m}$, coll. M. M. Bahir, A. Silva and K. Maduwage 24 IX 2004.

Hemipenis bilobed (divided for more than half of its length) (Fig. 1a). Length of entire organ greater than its width. Base naked (Fig. 1a). Sulcus spermaticus bifurcate, each branch of sulcus extending along each lobe, terminating at apex (Fig. 1a); proximal undivided half of sulcus spermaticus deep, distal half divided, shallow; lips of proximal half calyculate, lips of distal half smooth, widely open at apex (Fig. 1a). Calyculate ornamentation present on each lobe except proximal three-fourths of medial side of inner lobe (similarly on lateral side of outer lobe) (Fig. 1 $a, c$ ). Medial side of inner lobe and lateral side of outer lobe each with 12 flounces, the distal 7 flounces enlarged, pointed ventrally (Fig. 1a). Thin-walled, large, smooth calyces present on proximal half of dorsal and ventral sides of each lobe, forming deep polygonal pits (Fig. 1a, $c, d$ ). Thick-walled, small, smooth calyces present on distal half of dorsal and ventral sides of each lobe, forming shallow triradiate pits (Fig. 1b). Smooth flounces present medially on proximal half of ventral side of hemipenis (Fig. 1c). Apex divided into four segments: two similar-sized dorsal segments and two ventral segments (Fig. 1a). Apex naked.

\section{Otocryptis wiegmanni}

Material examined: WHT 6231. L, $70.2 \mathrm{~mm}$ SVL, Morningside Forest, Eastern Sinharaja, Sri Lanka, 06²4'N, 80³6' E, alt. 1080 m, coll. M. M. Bahir, A. Silva and K. Maduwage 10 XI 2004.

Hemipenes bilobed (divided for more than half its length) (Fig. 2a). Length of entire organ greater than its width. Base naked. Sulcus spermaticus bifurcate, each branch of sulcus extending along each lobe, terminating at apex (Fig. 2a); proximal undivided half of sulcus spermaticus deep, distal half divided, shallow; lips of proximal half calyculate, lips of distal half smooth, widely open at apex (Fig. 2a). Calyculate ornamentation present on each lobe except proximal three-fourths of medial side of inner lobe (as on lateral side of outer lobe) (Fig. $2 a$ and $c$ ). Medial side of inner lobe and lateral side of outer lobe each with 10 flounces, distal 3 flounces enlarged, pointed ventrally (Fig. $2 a, c$ ). Thin-walled, large, smooth calyces present on proximal half of dorsal and ventral sides of each lobe, forming deep polygonal pits (Fig. 2b). Thick-walled, small, smooth calyces present on distal half of dorsal and ventral sides of each lobe, forming shallow triradiate pits (Fig. 2b). Apex divided into four segments: two similarsized dorsal segments and two ventral segments (Fig. 2a). Apex naked.

\section{Sitana ponticeriana}

Material examined: WHT 7377, $41.9 \mathrm{~mm}$ SVL, Bundala, $06^{\circ} 11^{\prime} \mathrm{N}, 81^{\circ} 16^{\prime} \mathrm{E}$, alt. $5 \mathrm{~m}$, coll. A. Silva, T. Maduwage \& K. Maduwage 09 VIII 2006.

Hemipenis bilobed (divided for more than half of its length) (Fig. 3a). Length of entire organ greater than its width. Base naked. Sulcus spermaticus single (Fig. 3a). Lips smooth, widely open at apex. Calyculate ornamentation present on each lobe except proximal half of medial side of inner lobe (similarly on lateral side of outer lobe) (Fig. $3 a$ and $c$ ). Medial side of inner lobe with 6 flounces, lateral side of outer lobe with $\sim 12$ flounces (Fig. 3c). Thinwalled, large, smooth calyces present on proximal half of dorsal and ventral sides of each lobe, forming shallow polygonal calyces (Fig. $3 c$ ). Thick-walled, small, smooth calyces present on distal half of dorsal and ventral sides of each lobe, forming shallow triradiate pits (Fig. $3 b$ and c). Apex naked. 
$a$

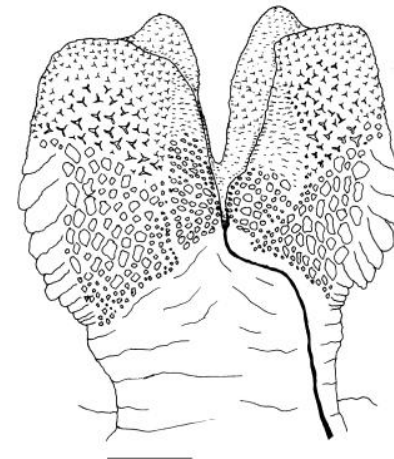

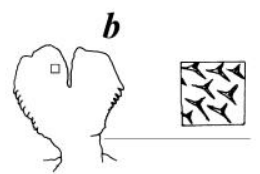
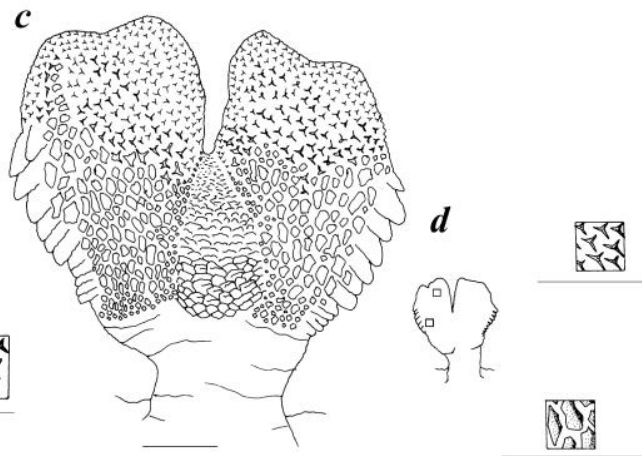

Figure 1.Otocryptis nigristigma: WHT 6168, $60.3 \mathrm{~mm} \mathrm{SVL,} a$, dorsal view; $b$, dorsal ornamentation; $c$, ventral view; $d$, ventral ornamentation. Scale bar: $1 \mathrm{~mm}$.

$\boldsymbol{a}$

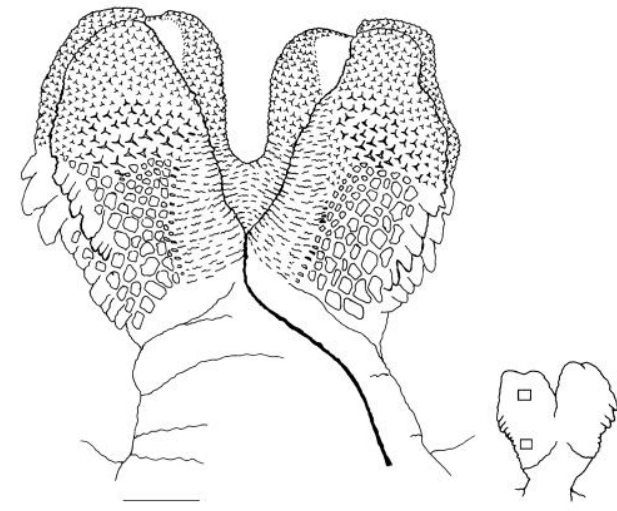

b

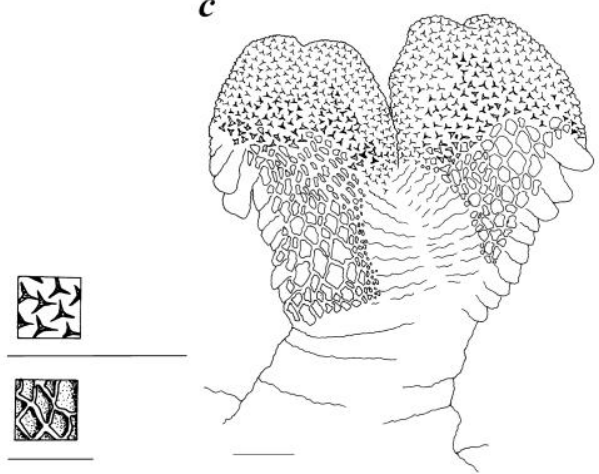

Figure 2. Otocryptis wiegmanni: WHT 6231.L, $70.2 \mathrm{~mm} \mathrm{SVL,} a$, dorsal view; $b$, dorsal ornamentation; $c$, ventral view. Scale bar: $1 \mathrm{~mm}$.

$a$

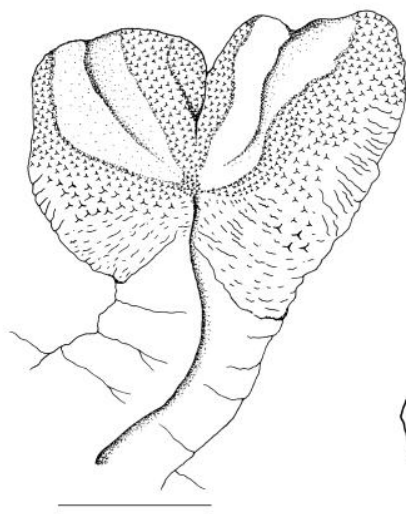

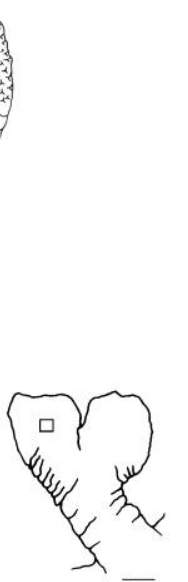

$c$

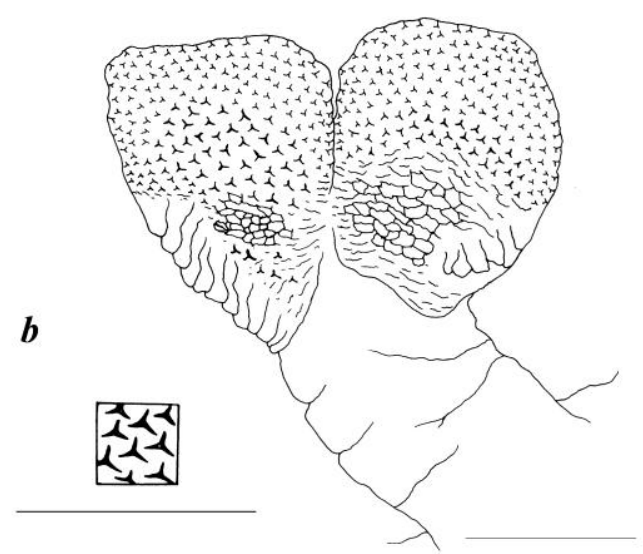

Figure 3. Sitana ponticeriana: WHT 7377, $41.9 \mathrm{~mm} \mathrm{SVL}, a$, dorsal view; $b$, ventral ornamentation; $c$, ventral view. Scale bar: $1 \mathrm{~mm}$. 
$a$

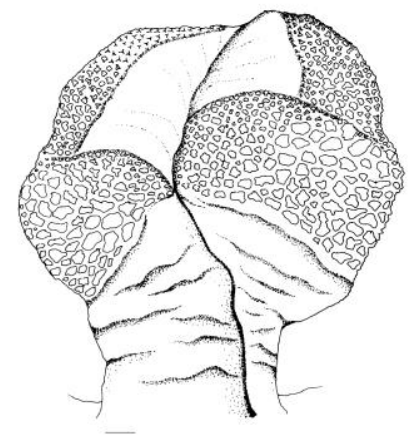

$c$

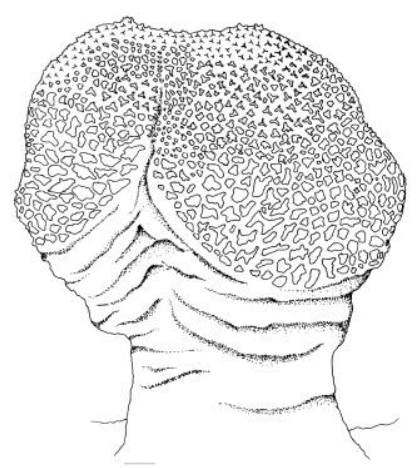

$b$
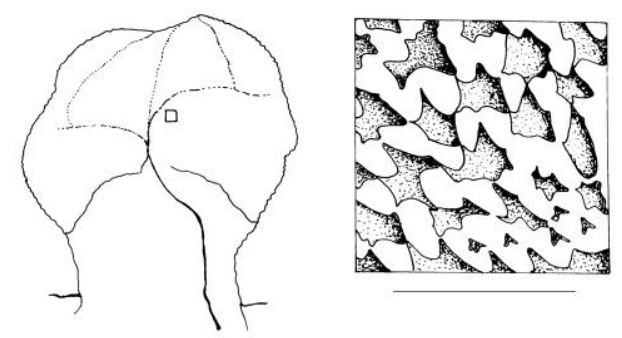

$d$

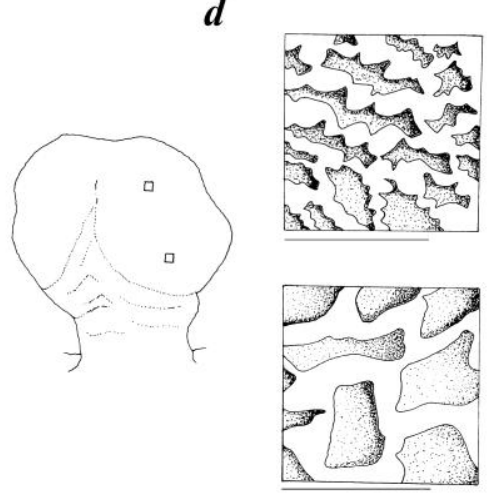

Figure 4. Lyriocephalus scutatus: WHT 6232, $111.4 \mathrm{~mm} \mathrm{SVL,} a$, dorsal view; $b$, dorsal ornamentation; $c$, ventral view; $d$, ventral ornamentation. Scale bar: $1 \mathrm{~mm}$.

\section{Lyriocephalus scutatus}

Material examined: WHT 6232, $111.4 \mathrm{~mm}$ SVL, Morningside Forest, Eastern Sinharaja, Sri Lanka, $06^{\circ} 24^{\prime} \mathrm{N}, 80^{\circ} 36^{\prime} \mathrm{E}$, alt. $1080 \mathrm{~m}$, coll. M. M. Bahir, A. Silva and K. Maduwage 10 XI 2004.

Hemipenis single, clavate (divided for less than half of its length) (Fig. $4 a$ and $c$ ). Length of entire organ less than its width (Fig. $4 c$ ). Base naked. Sulcus spermaticus single; proximal half of sulcus spermaticus deep, distal half shallow (Fig. 4a). Lips of sulcus spermaticus smooth, widely open at apex (Fig. 4a). Calyculate ornamentation present on each lobe (Fig. $4 a$ and c). Thick-walled, large, smooth calyces present on proximal half of ventral side of each lobe, forming deep polygonal pits (Fig. 4d). Thickwalled, minutely denticulated calyces present on distal half of dorsal and ventral sides of each lobe, forming shallow triradiate pits (Fig. 4d). Ventral sulcus smooth, not reaching apex (Fig. 4 c). Apex divided into four segments: two short dorsal segments and two long ventral segments (Fig. 4a). Apex naked.

\section{Cophotis ceylanica}

Material examined: WHT 2264, $59.8 \mathrm{~mm}$ SVL, Horton Plains National Park, 06²6’ N, 8047' E, alt. $2135 \mathrm{~m}$.

Hemipenis single, clavate (divided for less than half of its length) (Fig. 5a). Length of entire organ less than width. Base naked. Sulcus spermaticus single; proximal half of sulcus spermaticus deep, distal half shallow (Fig. 5a, $b)$. Lips of sulcus spermaticus smooth, widely open at apex (Fig. $5 a$ and $b$ ). Calyculate ornamentation present on each lobe (Fig. $5 a, b$ ). Thick-walled, large, averagely uniform in size, smooth calyces, forming deep polygonal pits (Fig. 5b). Ventral sulcus smooth, not reaching to the apex (Fig. $5 a$ and $b$ ). Apex divided into four segments: two short dorsal segments and two long ventral segments (Fig. $5 a, b$ ). Apex naked. 


\section{Ceratophora aspera (Fig. $6 a, b$ )}

Material examined: WHT 1401, $31.3 \mathrm{~mm}$ SVL, Kottawa proposed forest reserve, near Galle, $06^{\circ} 06^{\prime} \mathrm{N}, 80^{\circ} 20^{\prime} \mathrm{E}$, alt. $60 \mathrm{~m}$, coll. M. M. Bahir 03 III 1996.

Hemipenis single, clavate (divided for less than half of its length) (Fig. 6a). Length of entire organ greater than width. Base naked. Sulcus spermaticus single; proximal half of sulcus spermaticus deep, distal half shallow (Fig. 6a). Lips of sulcus spermaticus smooth, widely open at apex (Fig. 6a). Sulcus traverses apex (Fig. $6 a$ ). Calyculate ornamentation present on each lobe (Fig. $6 a$ and $b$ ). Smooth calyces, forming shallow polygonal pits (Fig. 6b). Apical calyces smaller than ventral and dorsal ones. Ventral sulcus wide, reaches to apex (Fig. 6b). Entire length of ventral sulcus with smooth transverse ridges (Fig. 6b). Lips of ventral sulcus with small, smooth calyces (Fig. 6b). Apex divided into four segments: two short dorsal segments and two long ventral segments (Fig. 6a). Apex naked. $\boldsymbol{a}$

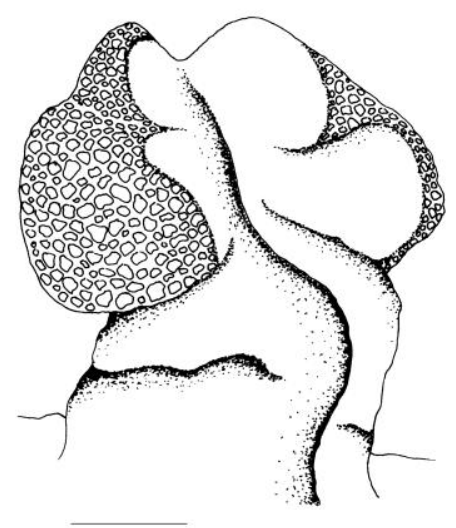

b

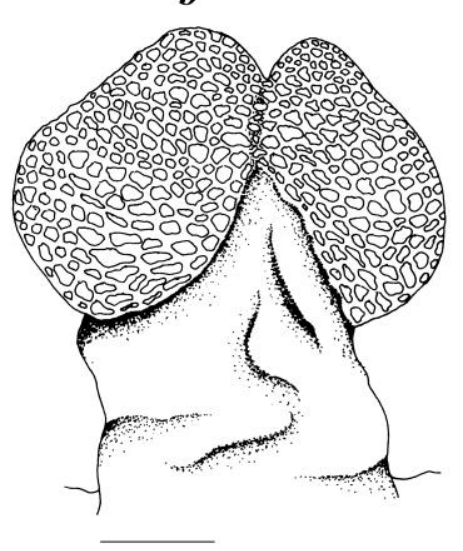

Figure 5. Cophotis ceylanica: WHT 2264, $59.8 \mathrm{~mm} \mathrm{SVL}$, $a$, dorsal view; $b$, ventral view. Scale bar: $1 \mathrm{~mm}$.
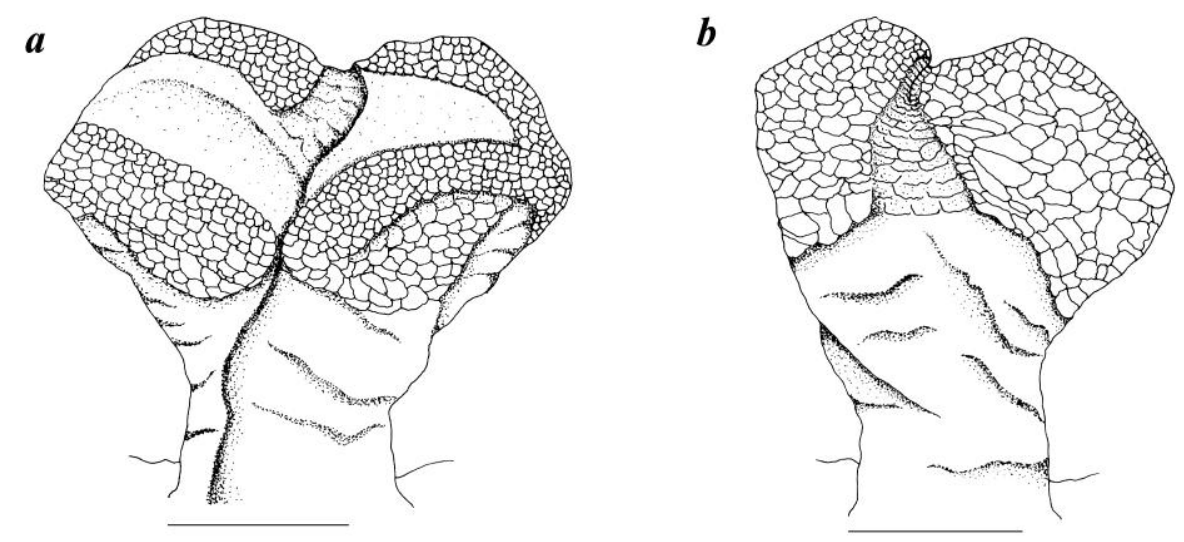

Figure 6. Ceratophora aspera: WHT 1401, $31.3 \mathrm{~mm} \mathrm{SVL,} a$, dorsal view; $b$, ventral view. Scale bar: 1 $\mathrm{mm}$. 


\section{Ceratophora erdeleni and C. karu}

Material examined: Ceratophora erdeleni; WHT 2070, $74.0 \mathrm{~mm}$ SVL, Morningside Forest, Eastern Sinharaja, Sri Lanka, 06² $4^{\prime} \mathrm{N}, 80^{\circ} 36^{\prime} \mathrm{E}$, alt. $1080 \mathrm{~m}$, coll. D. Gabadage 07 III 1996. C. karu; WHT 2066, 29.2 mm SVL, Morningside Forest, Eastern Sinharaja, Sri Lanka, 06 $24^{\prime} \mathrm{N}$, $80^{\circ} 36^{\prime}$ E, alt. $1080 \mathrm{~m}$, coll. D. Gabadage 07 III 1996.

Hemipenis single, clavate (divided for less than half of its length) (Figs. $7 a, 8 a$, respectively). Length of entire organ greater than the width. Base naked. Sulcus spermaticus single; proximal half of sulcus spermaticus deep, distal half shallow (Fig. 7a, 8a). Lips of sulcus spermaticus smooth, widely open at apex. Sulcus traverses apex (Fig. 7a, 8a). Calyculate ornamentation present on each lobe. Calyces smooth, forming shallow polygonal pits (Figs. $7 a, 7 b, 8 a$ and $8 b$ ). Apical calyces smaller than ventral and dorsal calyces. Ventral sulcus wide, reaching apex (Figs. $7 b$ and $8 b$ ). Distal one-third of ventral sulcus with smooth transverse ridges. Lips of ventral sulcus with small, smooth calyces. Apex divided into four segments: two short dorsal segments and two long ventral segments (Fig. $7 a$ and $8 a$ ). Apex naked. $a$

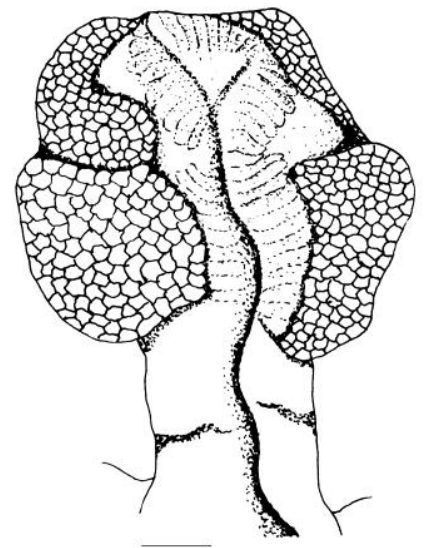

b

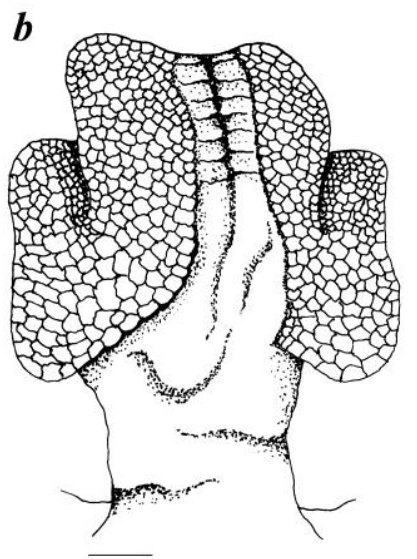

Figure 7: Ceratophora erdeleni: WHT 2070, $74.0 \mathrm{~mm} \mathrm{SVL,} a$, dorsal view; $b$, ventral view. Scale bar: 1 $\mathrm{mm}$.
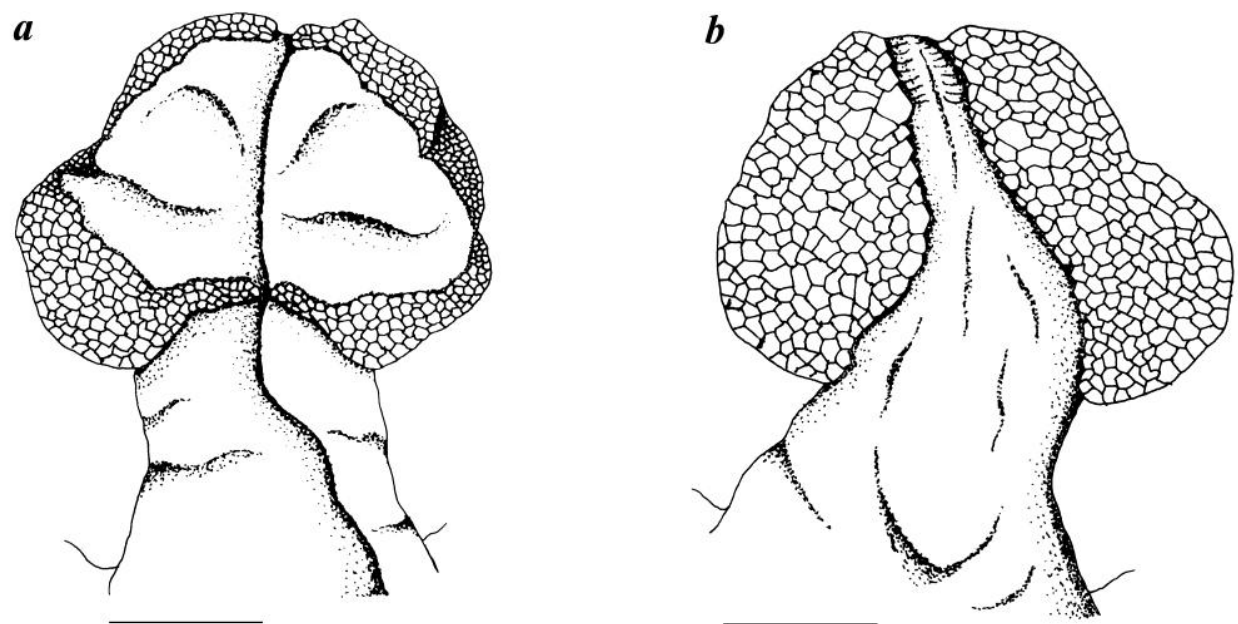

Figure 8. Ceratophora karu: WHT 2066, $29.2 \mathrm{~mm} \mathrm{SVL,} a$, dorsal view; $b$, ventral view. Scale bar: $1 \mathrm{~mm}$. 

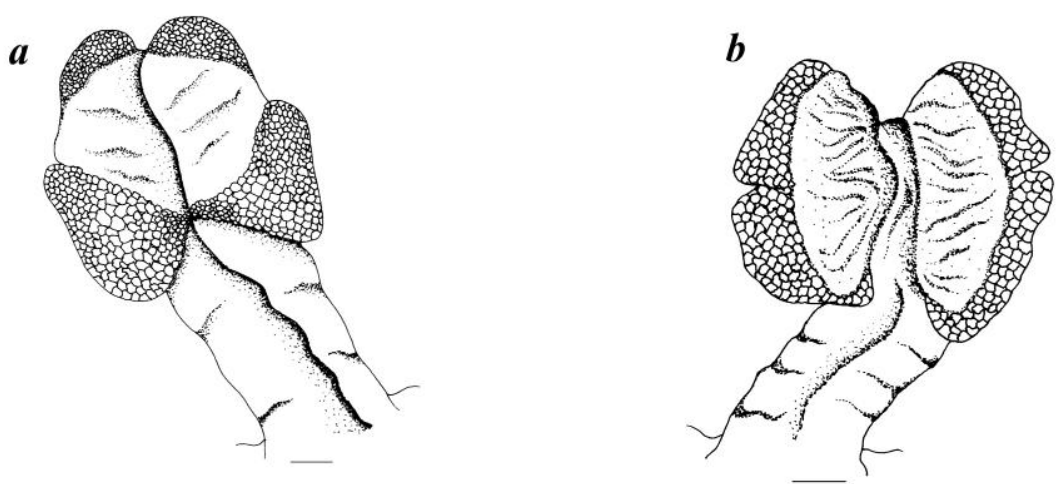

Figure 9. Ceratophora stoddartii: WHT 6501, $81.9 \mathrm{~mm}$ SVL, $a$, dorsal view; $b$, ventral view. Scale bar: 1 $\mathrm{mm}$
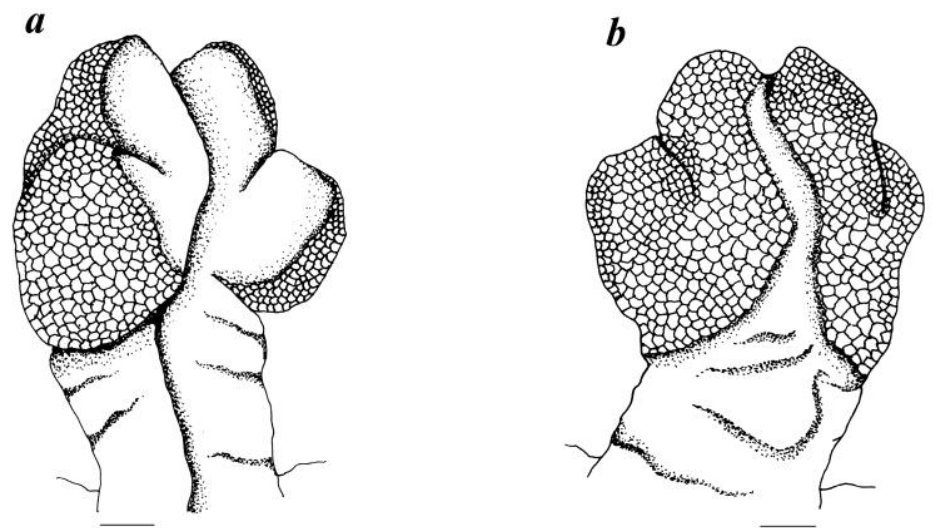

Figure 10. Ceratophora tennenti: WHT 7658, $71.2 \mathrm{~mm}$ SVL, $a$, dorsal view; $b$, ventral view. Scale bar: 1 $\mathrm{mm}$.

\section{Ceratophora stoddartii}

Material examined: WHT 6501, $81.9 \mathrm{~mm}$ SVL, Horton Plains National Park, 06 ${ }^{\circ} 46^{\prime} \mathrm{N}, 80^{\circ} 47^{\prime} \mathrm{E}$, alt. $2135 \mathrm{~m}$, coll. M. M. Bahir and S. V. Nanayakkara 23 VIII 2005.

Hemipenis, single, clavate organ (divided for less than half of its length) (Fig. 9a). Length of entire organ greater than width. Base naked. Sulcus spermaticus single; proximal half of sulcus spermaticus deep, distal half shallow (Fig. $9 a$ ). Lips of sulcus spermaticus smooth, widely open at apex (Fig. 9a). Sulcus traverses apex (Fig. 9a). Calyculate ornamentation present on each lobe. Smooth calyces, forming deep polygonal pits (Fig. $9 a$ and $b$ ). Calyces subequal along entire length of organ. Ventral sulcus wide, smooth, reaching apex. Lips of ventral sulcus wide, smooth. Apex divided into four segments: two short dorsal segments and two long ventral segments (Fig. $9 a$ and $b$ ). Apex naked.

\section{Ceratophora tennenti}

Material examined: WHT 7658 (formerly RFI 50399), 71.2 mm SVL, Corbett's Gap, Knuckles, $07^{\circ} 22^{\prime} \mathrm{N}, 80^{\circ} 51^{\prime} \mathrm{E}$, alt. $1100 \mathrm{~m}$, coll. M. M. Bahir and R. F. Inger 12 XI 2000.

Hemipenis single, clavate (divided for less than half its length) (Fig. $10 a$ and $b$ ). Length of entire organ greater than width. Base naked. Sulcus spermaticus single; proximal half of sulcus spermaticus deep, distal half shallow (Fig. 10a). Lips of sulcus spermaticus smooth, widely open at apex. Sulcus traverses apex (Fig. 10a). Calyculate ornamentation present on each lobe. Smooth calyces forming deep polygonal pits (Fig. $10 a$ and $b$ ). Apical calyces smaller than ventral and dorsal calyces. Ventral sulcus narrow, smooth, reaching apex. Lips of ventral sulcus with small, smooth calyces (Fig. 10a). Apex divided into four segments: two short dorsal segments and two long ventral segments (Fig. 10a). Apex naked. 
Calotes calotes and $\boldsymbol{C}$. versicolor

Material examined: Calotes calotes; WHT 6236, $113.0 \mathrm{~mm}$ SVL, Puwakpitiya, Knuckles, $07^{\circ} 34^{\prime} \mathrm{N}, 80^{\circ} 45^{\prime}$ E, alt. $450 \mathrm{~m}$, coll. M. M. Bahir, A. Silva and K. Maduwage 24 IX 2004. C. versicolor; WHT 7516, $94.0 \mathrm{~mm} \mathrm{SVL}$, Kanneliya Forest Reserve, near Udugama, $06^{\circ} 15^{\prime} \mathrm{N}, 80^{\circ} 20^{\prime} \mathrm{E}$, alt. $150 \mathrm{~m}$, coll. S. V. Nanayakkara 23 IX 2007.

Hemipenis single, clavate (divided for less than half of its length) (Figs. 11a, $c ; 12 a, c$ ). Length of entire organ greater than its width. Base naked. Sulcus spermaticus single; proximal twothirds of sulcus spermaticus deep, distal half shallow (Figs. 11a and 12a). Lips of sulcus spermaticus smooth, widely open at apex (Figs. $11 a$ and $12 a$ ). A fleshy cardioid structure present at base of ventral sulcus (Fig. 11b, $c ; 12 b, c$ ). Lateral and medial sulcus distinct only in upper half of each lobe. Calyculate ornamentation present on each lobe (Fig. 11a, $b, c ; 12 a, b, c)$. Thick-walled smooth calyces forming deep oval pits (Fig. 11b, $c ; 12 b, c)$. Apical calyces smaller than ventral and dorsal ones (Figs. 11a, $b, c$; $12 a, b, c)$. Ventral sulcus shallow in proximal two-thirds, deep in distal one-third, reaches apex (Fig. 11c, 12c). Proximal half of medial and lateral sulcus with smooth calyces (Figs. $11 b$ and $12 b$ ). Apex divided into four segments: two short dorsal segments and two long ventral segments by lateral and medial sulcus (Figs. $11 a$ and $12 a$ ). Apex naked.

\section{Calotes ceylonensis}

Material examined: WHT 1624, $65.7 \mathrm{~mm}$ SVL, Wasgamuwa, $07^{\circ} 40^{\prime} \mathrm{N}, 80^{\circ} 59^{\prime} \mathrm{E}$, alt. $50 \mathrm{~m}$, coll. M. M. Bahir 07 VI 1997.

Hemipenis single, clavate (divided for less than half of its length) (Fig. $13 a$ and $c$ ). Length of entire organ greater than its width. Base naked. Sulcus spermaticus single; proximal two-thirds of sulcus spermaticus deep, distal half shallow (Fig. 13a). Lips of sulcus spermaticus smooth, widely open at apex (Fig. 13a). A fleshy cardioid structure present at the base of ventral sulcus (Fig. $13 b$ and $c$ ). Lateral and medial sulcus distinct throughout length of each lobe (Fig. $13 b)$. Calyculate ornamentation present on each lobe (Fig. 13a, $b$ and $c$ ). Thick-walled smooth calyces forming deep oval pits (Fig. 13a, $b$ and $c$ ). Apical calyces smaller than ventral and dorsal calyces. Ventral sulcus shallow in proximal twothirds, deep in distal one-third, reaching apex (Fig. 13c). Entire length of medial and lateral sulcus with small, smooth calyces. Apex divided into four segments: two short dorsal segments and two long ventral segments by lateral and medial sulcus (Fig. 13a). Apex naked.
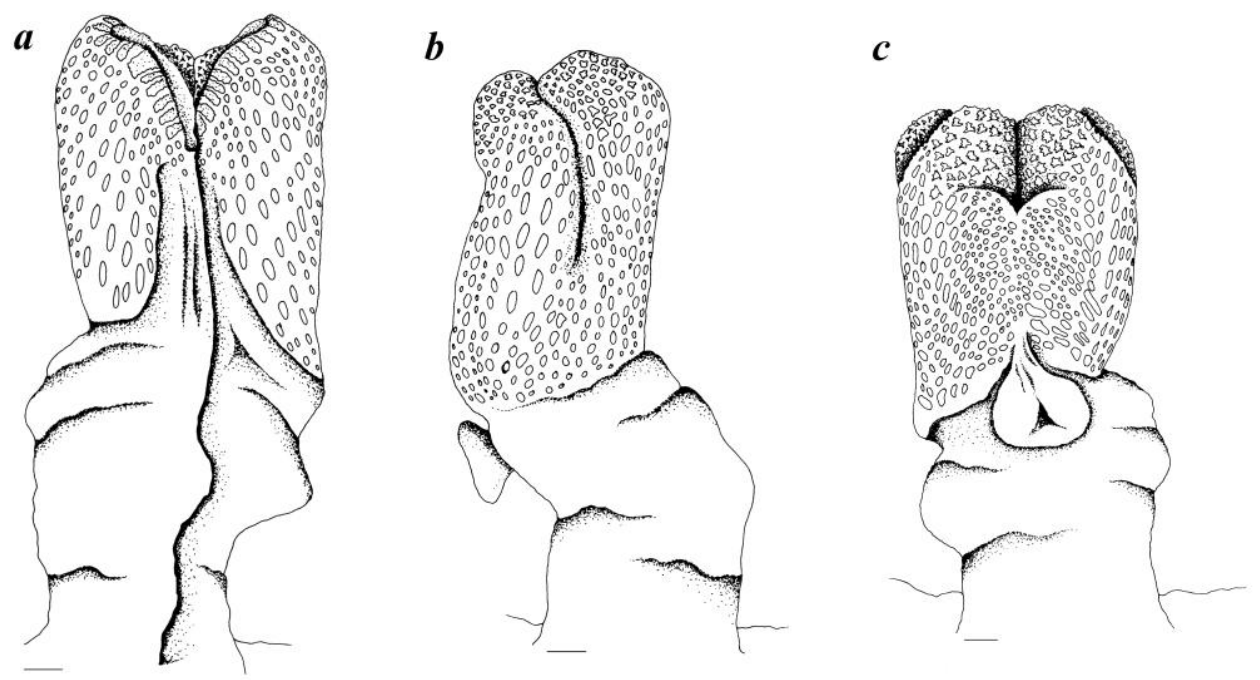

Figure 11. Calotes calotes: WHT 6236, $113.0 \mathrm{~mm} \mathrm{SVL}, a$, dorsal view; $b$, left lateral view; $c$, ventral view. Scale bar: $1 \mathrm{~mm}$. 
$a$

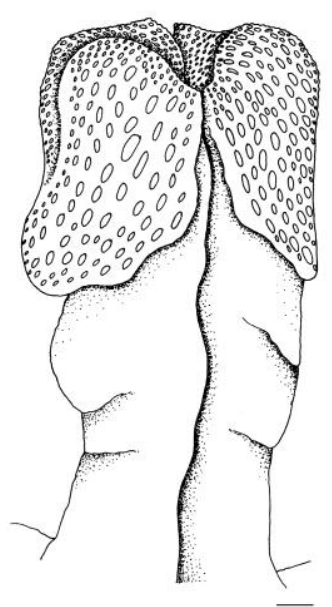

$b$

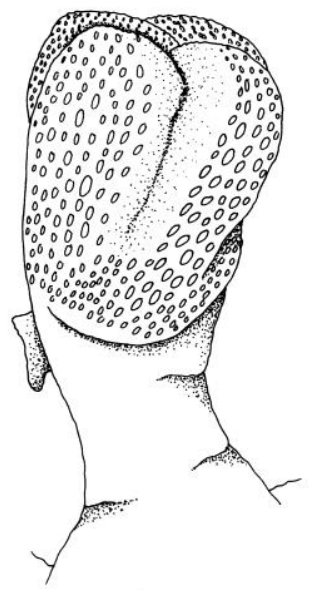

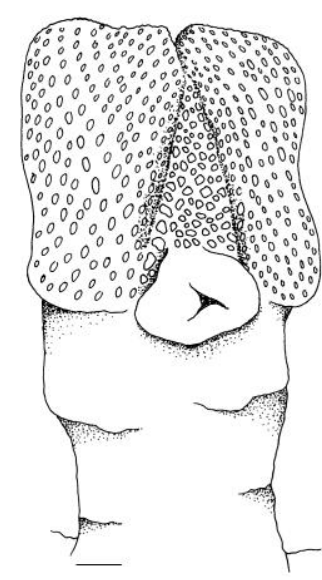

Figure 12. Calotes versicolor: WHT 7516, $94.0 \mathrm{~mm} \mathrm{SVL}, a$, dorsal view; $b$, lateral view; $c$, ventral view. Scale bar: $1 \mathrm{~mm}$.

\section{$a$}

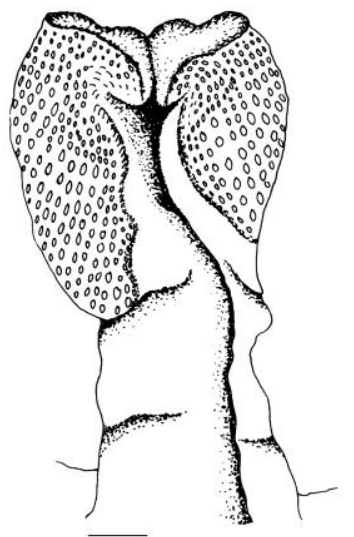

$b$

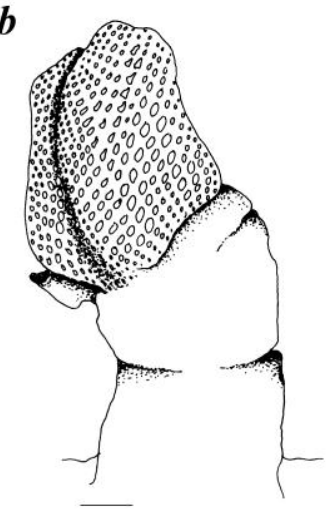

$c$

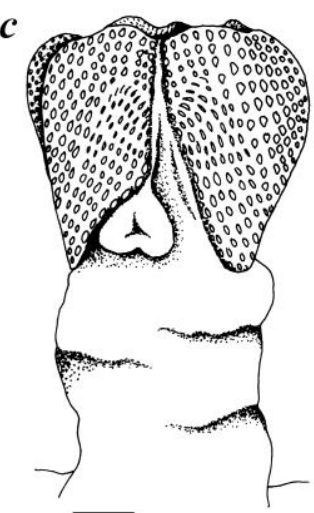

Figure 13. Calotes ceylonensis: WHT $1624,65.7 \mathrm{~mm} \mathrm{SVL}, a$, dorsal view; $b$, left lateral view; $c$, ventral view. Scale bar: $1 \mathrm{~mm}$.
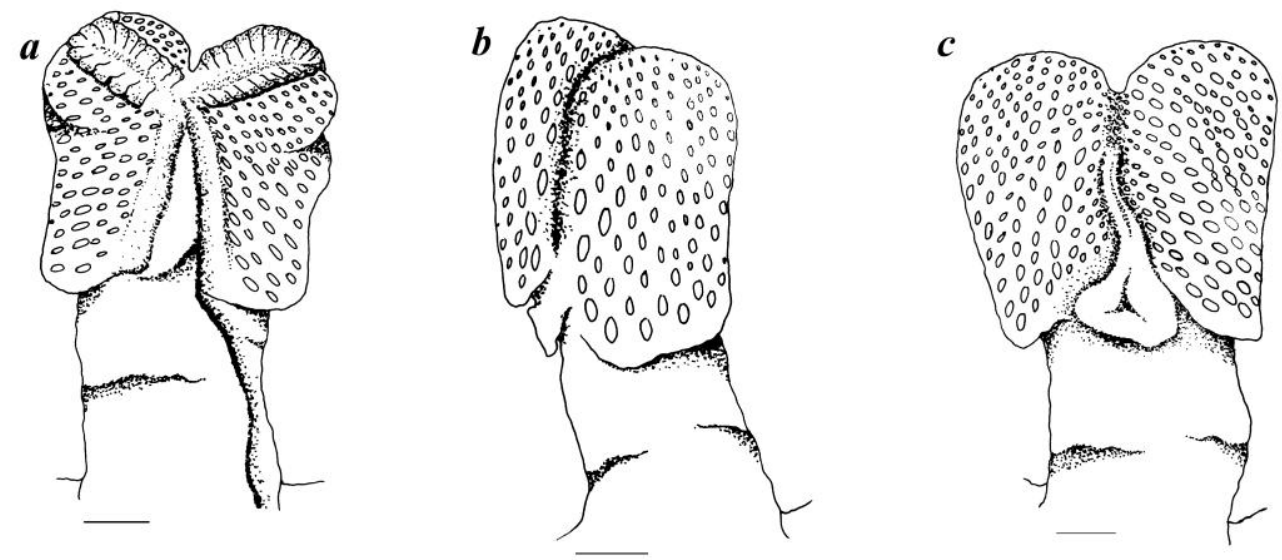

Figure 14. Calotes desilvai: WHT 503, $73.6 \mathrm{~mm} \mathrm{SVL,} a$, dorsal view; $b$, left lateral view; $c$, ventral view. Scale bar: $1 \mathrm{~mm}$. 
Calotes desilvai, C. liocephalus, C. liolepis and C. nigrilabris

Material examined: Calotes desilvai; WHT 0503, 73.6 mm SVL, Morningside Forest, Eastern Sinharaja, Sri Lanka, 06² $24^{\prime} \mathrm{N}, 80^{\circ} 36^{\prime} \mathrm{E}$, alt. $1080 \mathrm{~m}$, coll. M. M. Bahir and D. Gabadage 21 II 1996. C. liocephalus; WHT 6211, $90.2 \mathrm{~mm}$ SVL, Midland Estate, near Rattota, $07^{\circ} 31^{\prime} \mathrm{N}$, $80^{\circ} 44^{\prime}$ E, alt. 915 m, coll. M. M. Bahir, A. Silva and K. Maduwage 24 IX 2004. C. liolepis; WHT 6185, $90.5 \mathrm{~mm}$ SVL, Ritigala, $08^{\circ} 07^{\prime} \mathrm{N}, 80^{\circ} 40^{\prime}$ E, alt. 200 m, coll. M. M. Bahir, A. Silva and K. Maduwage 25 IX 2004. C. nigrilabris; WHT 0510, $85.9 \mathrm{~mm}$ SVL, Nuwara-Eliya, 06 $57^{\circ} \mathrm{N}$, $80^{\circ} 47^{\prime}$ E, alt. 1710 m, coll. M. M. Bahir and D. Gabadage 03 X 1995.

Hemipenis single, clavate (divided for less than half its length) (Fig. 14a, $b, c ; 15 a, b, c ; 16 a, b$, $c ; 17 a, b, c)$. Length of entire organ greater than its width. Base naked. Sulcus spermaticus single; proximal two-thirds of sulcus spermaticus deep, distal half shallow (Fig. 14a, 15a, 16a, 17a). Lips of sulcus spermaticus smooth, widely open at apex (Fig. 14a, 15a, 16a, 17a). A fleshy cardioid structure present at base of ventral sulcus (Fig. 14b, c, 15b, c, 16b, c, 17b, c). Lateral and medial sulcus distinct only in upper three-fourths of each lobe (Fig. 14b, 15b, 16b, $17 b)$. Calyculate ornamentation present on each lobe (Fig. 14a, $b, c ; 15 a, b, c ; 16 a, b, c ; 17 a, b$, $c$ ). Thick-walled smooth calyces forming deep oval pits (Fig. 14a, b, $c ; 15 a, b, c ; 16 a, b, c ; 17 a$, $b, c)$. Apical calyces smaller than ventral and dorsal calyces. Ventral sulcus shallow in proximal two-thirds, deep in distal one-third, reaching apex (Fig. 14c, 15c, 16c, 17c). Calyces absent in medial and lateral sulcus. Apex divided into four segments: two short dorsal segments and two long ventral segments, by lateral and medial sulcus (Fig. 14a, 15a, 16a, 17a). Apex naked.
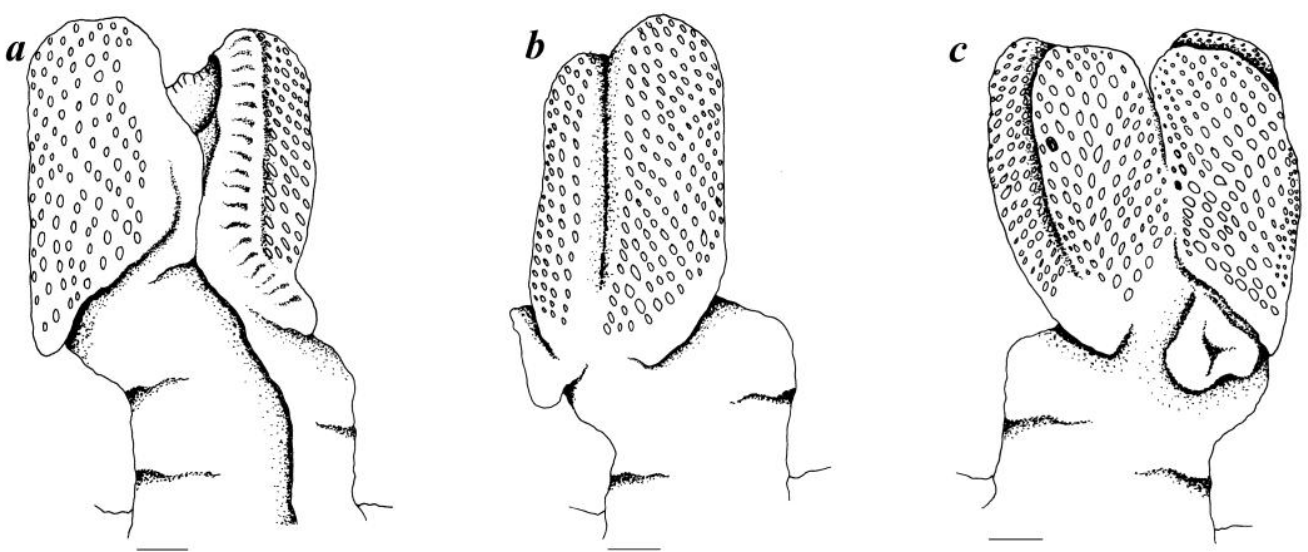

Figure 15. Calotes liocephalus: WHT 6211, $90.2 \mathrm{~mm} \mathrm{SVL,} a$, dorsal view; $b$, left lateral view; $c$, ventral view. Scale bar: $1 \mathrm{~mm}$.

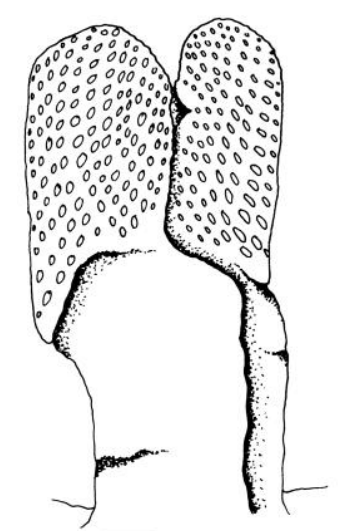

$\boldsymbol{b}$

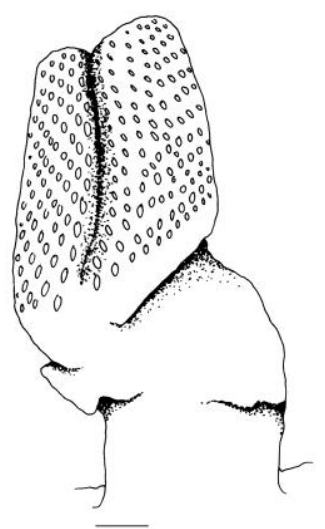

c

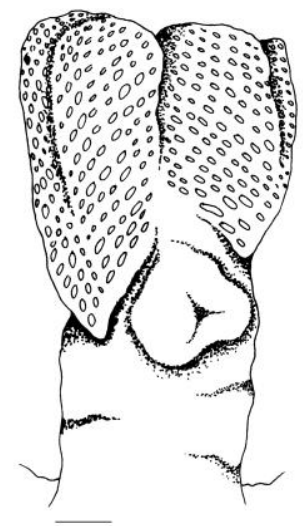

Figure 16. Calotes liolepis: WHT 6185, $90.5 \mathrm{~mm} \mathrm{SVL,} a$, dorsal view; $b$, left lateral view; $c$, ventral view. Scale bar: $1 \mathrm{~mm}$. 

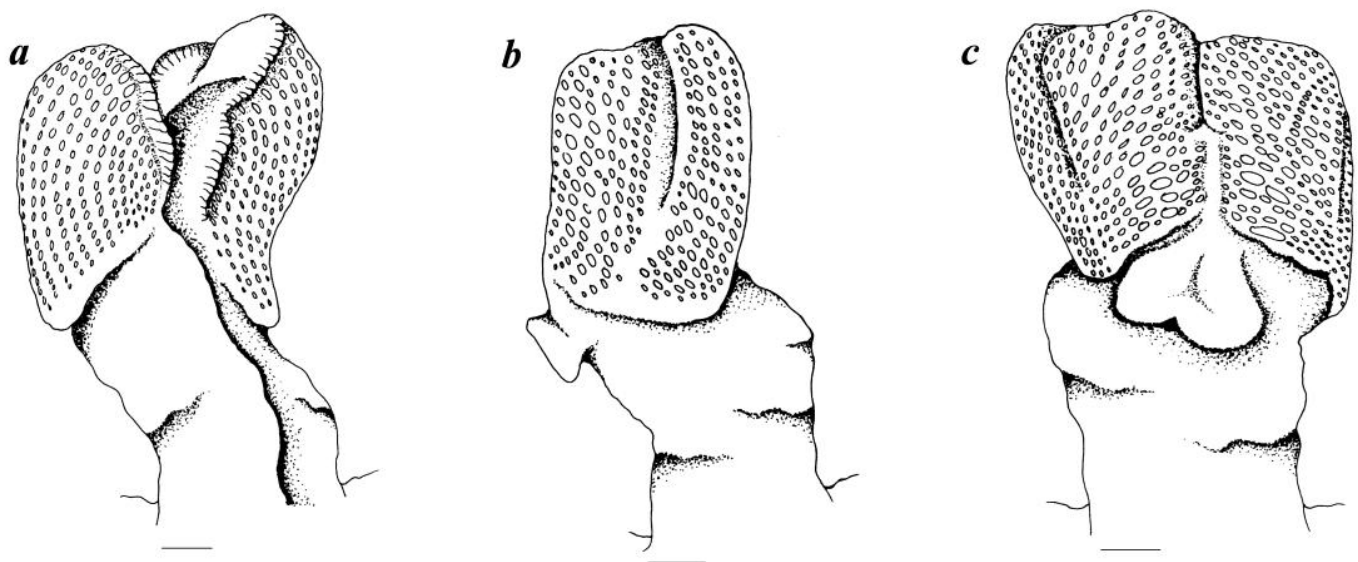

Figure 17. Calotes nigrilabris: WHT 0510, $85.9 \mathrm{~mm}$ SVL, $a$, dorsal view; $b$, left lateral view; $c$, ventral view. Scale bar: $1 \mathrm{~mm}$.

\section{Key to Sri Lankan Agamidae based on hemipeneal characters}

1. a. hemipenis divided for more than half its length, flounces present............................2

b. hemipenis divided for less than half its length, flounces absent................................

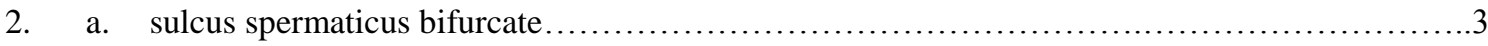

b. sulcus spermaticus single............................................. Sitana ponticeriana

3. a. each lobe with more than 11 flounces................................ Otocryptis nigristigma

b. each lobe with less than 11 flounces.............................. Otocryptis wiegmanni

4. a. a fleshy cardioid structure absent at base of ventral sulcus ....................................

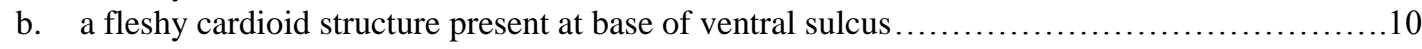

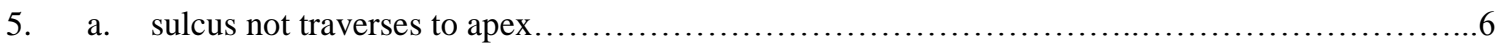

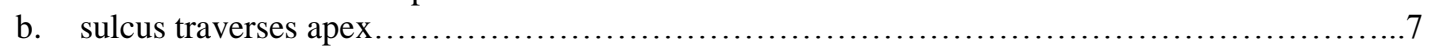

6 a. minute denticulation present on calyces............................ Lyriocephalus scutatus

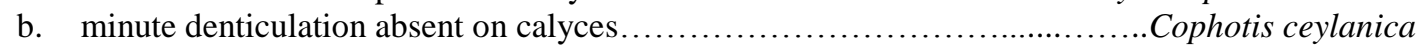

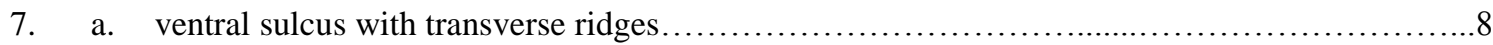

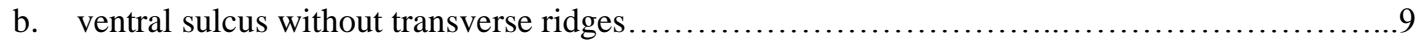

8. a. transverse ridges present on entire length of ventral sulcus....

Ceratophora aspera

b. transverse ridges present less than half length of ventral sulcus...............Ceratophora erdeleni and $C$. karu

9. a. Calyces subequal along the entire length of the organ.

Ceratophora stoddartii

b. apical calyces smaller then ventral and dorsal calyces

Ceratophora tennenti

10. a. calyces present in lateral and medial sulcus

.11

b. calyces absent in lateral and medial sulcus

Calotes liolepis, C. liocephalus,

C. nigrilabris and C. desilvai

11 a. Entire length of lateral and medial sulcus with calyces....

.Calotes ceylonensis

b. Calyces present only in proximal half of medial and lateral sulcus. 


\section{DISCUSSION}

The terminology and the characters used in hemipeneal studies may differ among various families of Sauria. Although those used by Dowling and Savage (1960) for snake hemipenes could be adapted to Agamids, they cannot be extended to families such as the Lacertidae and Chamaeleonidae owing to marked differences in both the overall structure and the micro-ornamentation of the hemipenes.

It is important to note that the characterization of the morphology of the everted hemipenis depends very much on the method of eversion (an incompletely everted hemipenis may lead to mischaracterization: Dowling and Savage, 1960). The hemipenes of all our specimens were everted by ourselves, for which reason we expect our observations to be consistent across species. We agree with Inger and Marx (1962), however, that intraspecific variation of the associated characters needs to be assessed so as to avoid awarding them undue taxonomic weight. However, the paucity of series of Sri Lankan agamid males with everted hemipenes limited our ability to assess variation within every species.

Synapomorphic hemipeneal character status has previously been described in Chamaeleonidae (Klaver and Böhme, 1986) and Lacertidae (Arnold, 1986). The present observations show that the Sri Lankan Agamidae too, possess hemipeneal characters relevant to their generic and suprageneric relationships. At the subfamily level, the shared characters are much broader, (e.g. habitus of the organ; hemipenis bilobed in Sitaninae vs. single or clavate in other subfamilies). At the generic level, most agamid genera in Sri Lanka display synapomorphic hemipeneal characters, (e.g. all species in the endemic genus Ceratophora have the sulcus traversing the hemipeneal apex). Within some genera, however, there is little interspecific variation in hemipeneal morphology (e.g. the endemic species of Calotes), whereas the various species of Otocryptis show substantial differences in hemipeneal morphology.

Clearly, an important (arguably the primary) function of the ornamentation observed in these hemipenes is to hold the intromittent organ in place in the female's cloaca until sperm could successfully be transferred. Why then the marked interspecific variation in hemipeneal morphology? This question was addressed in part by Böhme and Ziegler (2009), who observed: "peculiarities of shape and structure which do not seem to be functionally correlated [are] therefore considered to contain valuable phylogenetic signals". This indeed appears to be the case. However, the correlation they observed between low hemipenial diversity and marked, sexually dimorphic, epigamic characters in iguanians, versus high hemipenial diversity though almost entirely lacking sexual dimorphism in varanoids does not appear to hold true for the Sri Lankan agamids. This question deserves further investigation, as does the search for correlations between genital morphology and reproductive behaviour.

\section{ACKNOWLEDGEMENTS}

We thank Sudath Nanayakkara for the hospitality provided while visiting the WHT's field station at Agrapatana, Dharmasri Kandamby (National Maritime Museum, Galle) for his encouragements and Rohan Pethiyagoda for assistance in preparing the manuscript. We are grateful to two anonymous reviewers for comments that helped substantially to improve this manuscript.

\section{REFERENCES}

Arnold, E. N. (1986). The hemipenis of lacertid lizards (Reptilia: Lacertidae): structure, variation and systematic implications. Journal of Natural History 20(5): 12211257.

Bahir, M. M. and Silva, A. (2005). Otocryptis nigristigma, A new species of Agamid lizard from Sri Lanka. In: D. C. J. Yeo, P. K. L. Ng and R. Pethiyagoda (eds.), Contributions to biodiversity exploration and research in Sri Lanka. The Raffles Bulletin of Zoology, Supplement 12: 393-406.

Bahir, M. M. and Surasinghe, T. D. (2005). A conservation assessment of the Sri Lankan Agamidae (Reptilia: Sauria). In: Yeo, D. C. J., P. K. L. Ng and R. Pethiyagoda (eds.), Contributions to biodiversity exploration and research in Sri Lanka. The Raffles Bulletin of Zoology, Supplement 12: 407-412.

Baig, K. J. and Böhme, W. (1997). Partition of the "Stellio" group of Agama into two distinct genera: Acanthocercus FITZINGER 1843, and Laudakia GRAY 1845 (Sauria: Agamidae). In: W. Bischoff; W. Böhme. and T. Ziegler (Eds.) Herpetologia Bonnensis, Bonn (SEH). 
Böhme, W. and Ziegler, T. (2009). A review of iguanian and anguimorph lizard genitalia (Squamata: Chamaeleonidae; Varanoidea, Shinisauridae, Xenosauridae, Anguidae) and their phylogenetic significance: comparisons with molecular data sets. Journal of Zoological Systematics and Evolutionary Research 47(2): 189-202.

Branch, W. R. (1982). Hemipeneal morphology of Platynotan lizards. Journal of Herpetology 16: 16-38.

Cope, E. D. (1894). The classification of snakes. American Naturalist 28 (334): 831-844.

Cope, E. D. (1895). The classification of Ophidia, Transactions of the American Philosophical Society. 28: 186-219.

Cope, E. D. (1896). On the hemipenes of the Sauria. Proceedings of The Academy of Natural Sciences Philadelphia 48: 461-467.

Dowling, H. G. and Savage, J. M. (1960). A guide to the snake hemipenis: a survey of basic structure and systematic characteristics. Zoologica 45: 17-28.

Inger, R.F. and Marx, H. (1962). Variation of hemipenis and cloaca in the colubrid snake, Calamaria lumbricoidea. Systematic Zoology 11: $32-38$.

Klaver, C. and Böhme, W. (1986). Phylogeny and classification of the Chamaeleonidae (Sauria) with special reference to hemipenis morphology. Bonner Zoologische Monographien 22: 1-64.

Köehler, J., Hahn, M and Köhler, G. (2012). Divergent evolution of hemipenial morphology in two cryptic species of mainland anoles related to Anolis polylepis. Salamandra 48(1): 1-11.

Maduwage, K. P., Meegaskumbura, M., Silva, A. and Pethiyagoda, R. (2008). Phylogenetic utility of hemipeneal morphology in Sri Lankan agamid lizards. Current Science 95(7): 838-840.
McCann, C. (1946). The hemipenis in reptiles. Journal of Bombay Natural History Society 46(2): 348-373, pls 1-10.

Pope, C. H. and Pope, S. H. (1933). A study of the green pitvipers of southeastern Asia and Malaysia, commonly identified as Trimeresurus gramineus, Shaw, with a description of a new species from Peninsular India. American Museum Novitates 620: 1-12.

Presch, W. (1978). Descriptions of the Hemipenial Morphology in Eight Species of Microteiid Lizards (Family Teiidae, Subfamily Gymnophthalminae). Herpetologica 34(1): 108112.

Samarawickrama, V. A. M. P. K., Ranawana, K. B., Rajapaksha, D. R. N. S., Ananjeva, N. B., Orlov, N. L., Ranasinghe, J. M. A. S. and Samarawickrama, V. A. P. (2006). A new species of the genus Cophotis (Squamata: Agamidae) from Sri Lanka. Russian Journal of Herpetology 13(3): 207-214.

Semenov, D. V. and Dunaev, E. A. (1989). Morphology of the Hemipenis and classification of Lizards of the Genus Phrynocephalus; Reptilis, Agamidae. Zoologicheskii Zhurnal 68(10): 56-64.

Smith, M. A. (1943). Reptilia and Amphibia. Vol. 3: Serpents, in the fauna of British India, Ceylon and Burma, including the entire of the IndoChinese sub-region. Taylor and Francis, London, England. xii +583 pp.

Smith, H. M. and Fritts, T. H. (1969). Cannibalism in the Lizard Sceloporus chrysostictus. Journal of Herpetology 3 (3/4): 182-183.

Wall, F. (1923). A review of the Indian species of the Genus Oligodon suppressing the genus Slmotes (Ophidia) Calcutta. Records Indian Museum 25: 305-334. 